\title{
Mammalian and avian diversity of the Rewa Head, Rupununi, Southern Guyana
}

\author{
Robert Stuart Alexander Pickles ${ }^{1,2}$, Niall Patrick McCann ${ }^{1}$ \& Ashley Peregrine Holland ${ }^{1}$ \\ ${ }^{1}$ Institute of Zoology, Zoological Society of London, Regent's Park, London, NW1 4RY, \\ School of Biosciences, Cardiff University, Museum Avenue, Cardiff, Wales, \\ CF103AX Rupununi River Drifters, Karanambu Ranch, Lethem Post Office, Region 9, Rupununi Guyana \\ ${ }^{2}$ Corresponding author: Robert Stuart Alexander Pickles,e-mail: robert.pickles@ioz.ac.uk
}

PICKLES, R.S.A., McCANN, N.P. \& HOLLAND, A.L. Mammalian and avian diversity of the Rewa Head, Rupununi, Southern Guyana. Biota Neotrop. 11(3): http://www.biotaneotropica.org.br/v11n3/en/abstract?in ventory+bn00911032011

\begin{abstract}
We report the results of a short expedition to the remote headwaters of the River Rewa, a tributary of the River Essequibo in the Rupununi, Southern Guyana. We used a combination of camera trapping, mist netting and spot count surveys to document the mammalian and avian diversity found in the region. We recorded a total of 33 mammal species including all 8 of Guyana's monkey species as well as threatened species such as lowland tapir (Tapirus terrestris), giant otter (Pteronura brasiliensis) and bush dog (Speothos venaticus). We recorded a minimum population size of 35 giant otters in five packs along the $95 \mathrm{~km}$ of river surveyed. In total we observed 193 bird species from 47 families. With the inclusion of Smithsonian Institution data from 2006, the bird species list for the Rewa Head rises to 250 from 54 families. These include 10 Guiana Shield endemics and two species recorded as rare throughout their ranges: the harpy eagle (Harpia harpyja) and crested eagle (Morphnus guianensis).

Keywords: Guiana Shield, biodiversity conservation, endemic and threatened birds, endangered species of mammal, Rewa River, Rupununi.
\end{abstract}

PICKLES, R.S.A., McCANN, N.P. \& HOLLAND, A.L. Diversidade de mamíferos e aves do Chefe Rewa, Rupununi, Guiana do Sul. Biota Neotrop. 11(3): http://www.biotaneotropica.org.br/v11n3/pt/abstract?invent ory+bn00911032011

Resumo: Reportamos os resultados de uma curta expedição às remotas cabeceiras do Rio Rewa, tributário do Rio Essequibo no interior da Guiana. Utilizamos uma combinação de armadilhas fotográficas, redes de neblina e avistamento para documentar a diversidade de mamíferos e aves encontrados na região. Nós registramos um total de 33 espécies de mamíferos, incluindo todas as oito espécies de primatas que ocorrem na Guyana, além de espécies ameaçadas como anta (Tapirus terrestris), ariranha (Pteronura brasiliensis) e cachorro-do-mato (Speothos venaticus). Nós registramos uma população mínima de 35 ariranhas em cinco grupos no $95 \mathrm{~km}$ do rio. Registramos também um total de 187 espécies de aves que pertencem a 47 diferentes famílias. Incluindo os dados gerados pelo Smithsonian Institution em 2006, a lista de espécies para a cabeceira do Rio Rewa aumenta para 252, com 48 famílias, incluindo 10 espécies endêmicas do escudo das Guianas e duas espécies consideradas raras: a harpia (Harpia harpyja) e o gavião-real-falso (Morphnus guianensis).

Palavras-chave: Escuda da Guiana, conservação da biodiversidade, endêmicas e ameaçadas de aves, espécies ameaçadas de mamíferos, Rio do Rewa, Rupununi. 


\section{Introduction}

The Guiana Shield comprises one of the largest single tracts of forest anywhere in the tropics, spanning 250 million ha, and is consequently of high importance for a range of lowland rainforest species (Hammond 2005). Over 20,000 species of vascular plants are found in the Guiana Shield ecoregion, $35 \%$ of which are endemic. Similarly 975 bird species are found in this region, of which over 70 are endemic (Hammond 2005). Guyana itself contains 812 species of bird (Milensky et al. 2005). Our understanding of the diversity and distribution of mammal species in Guyana is patchy and is derived mainly from studies conducted in Iwokrama Forest. So far 225 species of mammal have been identified in Guyana, 146 of which are bats (Engstrom \& Lim 2008), and of which 29 are classified as threatened (Lim \& Engstrom 2004). Of the mammals found in Guyana, 29 are classified as threatened (IUCN 2008). Furthermore, the Guiana Shield contains some of the most carbon-rich forests in South America and represents an important carbon dioxide sink (Saatchi et al. 2007).

Our expedition in January 2009 followed on from a Conservation International Rapid Assessment Programme (RAP) in the Eastern Kanuku Mountains and Lower Kwitaro River in 2001 (Montambaut \& Missa 2002), and a Smithsonian Institution expedition exploring the avifauna of the River Rewa, which sampled two sites in the Rewa Head and a further site below Corona Falls (Milensky \& Schmidt 2006). A second Conservation International RAP in 2006, explored the biodiversity of the Konashen Community Owned Conservation Area (COCA) in southern Guyana (Alonso et al. 2006). Apart from these few short expeditions, the biodiversity of the Upper Takutu-Upper Essequibo Region is remarkably unexplored.

Lying between 1 and 9 degrees north of the Equator with a coast in the Caribbean, Guyana's weather patterns are driven by the Caribbean Intertropical Convergence Zone, with the rainy season arriving in early May lasting until mid-August, followed by another short rainy season in December. Its forests are hot and humid with between 2000-4000 mm of rain annually (LOC 2002). Guyana has a small population of 751,000 , with a population density of 3.5 per $\mathrm{km}^{2} .90 \%$ of the population live in a strip of land around the cities of Georgetown, Bartica and Linden in the north of the country (Beaie 2002). Guyana has never had government-led drives to open up the interior of the country to commercial development, consequently its forests have remained largely intact and land cover remains 76\% rainforest (Guyana Forestry Commission 2007).

The focal species of the expedition, the giant otter, is listed as 'Endangered' under the IUCN Red List due to overhunting during the last century (Duplaix et al. 2008). Following the listing of the giant otter under the Convention on the International Trade in Endangered Species (CITES) and the collapse of the international market for carnivore skins, the species has begun to recover in some areas. However, populations remain fragmented, and although the threat of commercial hunting has declined, habitat degradation such as gold dredging has increased (Groenendijk et al. 2005). Guyana was considered to be one of the strongholds for the giant otter due to the low human density of the country, and the number of inaccessible creeks. Hunting did occur throughout the Rupununi, but the population along this major tributary of the Essequibo was never extirpated (McTurk pers. comm.). Interviews with local fishermen who previously commercially hunted giant otter report that fur hunters never ventured above Corona Falls. The river may therefore provide good baseline data for healthy giant otter population densities.

\section{Situation of the Rewa Head}

The Rewa Head is located in Southern Guyana, in Upper Takutu-Upper Essequibo Administrative Region (Figure 1). It takes its water from tributaries feeding from the Kanuku Mountains in the South and drains north into the Rupununi and Essequibo before flowing into the Atlantic. The Rewa is a blackwater river due to the humic, yet relatively sediment-free waters. Following the Rewa upstream from where it is met by the Kwitaro, the lowland rainforest vegetation type continues up above Corona Falls. Above here the river is fractured by a series of cataracts and falls which prevent the colonisation of the headwaters by fish common in the Lower Rewa, such as arapaima (Arapaima gigas), lukanani (Cichla ocellaris), arawana (Osteoglossum bicirrhosum). Whereas black caiman (Melanosuchus niger), spectacled caiman (Caiman crocodilus) and the giant Amazonian river turtle (Podocnemis expansa) are found in the Lower Rewa up to the base of Corona Falls, none of these species were recorded in the Rewa Head. A series of narrow tributaries flow into the Rewa along its meandering path above the falls. Continuing upstream, the river narrows to $6 \mathrm{~m}$ wide by $2^{\circ} 45.358^{\prime} \mathrm{N}$ and $58^{\circ}$ $37.415^{\prime} \mathrm{W}$ and shortly after, at $2^{\circ} 45^{\prime} \mathrm{N}$ and $58^{\circ} 33^{\prime} \mathrm{W}$ the vegetation becomes scrubby riparian bush with dense bamboo groves, cecropia and guava, continuing with patchy forest to $2^{\circ} 42^{\prime} \mathrm{N}$ where dense forest once again predominates.

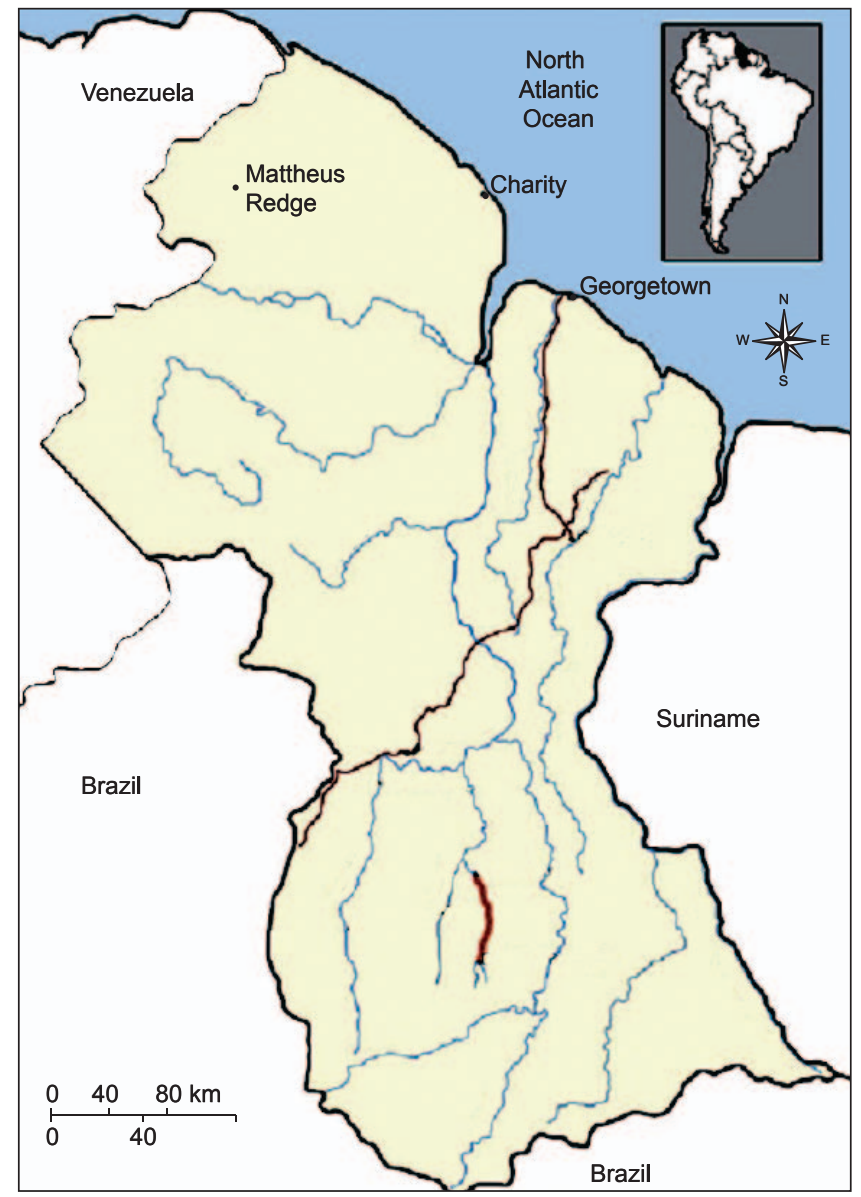

Figure 1. Location of the Rewa Head and extent surveyed by the expedition (highlighted in red).

Figura 1. Localização da cabeceira do Rio Rewa e extensão da área estudada durante a expedição (em vermelho). 


\section{Specific objectives}

The aims of the expedition were to (1) record the diversity of birds and large mammals along a lowland rainforest riparian corridor of very low disturbance in Guyana's interior and (2) to estimate the size of the giant otter population along the focal river. The Rewa Head was selected due to its remote situation and the likelihood of encountering undisturbed forest communities. In adding to our knowledge of the species richness and composition in this region, it was also our objective to increase scientific attention in a largely neglected yet potentially highly diverse section of the Guianas.

\section{Materials and Methods}

The headwaters of the River Rewa, the 'Rewa Head' were explored from Corona Falls at $3^{\circ} 10^{\prime} 34^{\prime \prime} \mathrm{N}$ and $58^{\circ} 40^{\prime}$ ' 26" W for 95 $\mathrm{km}$ up to the East-West Rewa split at $2^{\circ} 37^{\prime} 45^{\prime \prime} \mathrm{N}$ and $58^{\circ} 37^{\prime}$ '9" W where the river ceased to be navigable due to fallen logs (Figure 1). The expedition ran from the $31^{\text {st }}$ December 2008 to the $31^{\text {st }}$ January 2009 during the period of low water before the onset of the winter rains. Two $7 \mathrm{~m}$ heavy duty aluminium boats with $15 \mathrm{hp}$ outboard engines were used. Positions of camps where mist-netting surveys took place are given in Appendix 1.

\section{Camera trapping}

Twelve Reconyx RC55 camera traps were set up along the River Rewa above Corona Falls. Each trap was fixed to a tree or stake approximately $50 \mathrm{~cm}$ above the ground. The traps were set up in pairs, with one on the river bank itself facing inland and its partner $150 \mathrm{~m}$ perpendicular to the river bank facing a direction estimated to best increase the likelihood of capture. The pairs were arranged $8 \mathrm{~km}$ apart and left for a maximum of 22 days before collection. Camera traps were situated in open ground where an unobstructed view of the forest floor ensured the maximum potential for capture success. Due to the strict adherence of the $8 \mathrm{~km} / 150 \mathrm{~m}$ rule, we ensured that to some extent the placement of the traps was randomised and took in a variety of micro-habitats from dense scrubby marshland to hill tops to open riparian bush. The Relative Abundance Index (RAI) of mammalian species recorded was calculated and each camera was considered a separate sampling site.

\section{River surveying}

In conducting drift spot count surveys, the Rewa Head was divided into $8 \mathrm{~km}$ stretches, with each stretch surveyed once while drifting downstream. The survey boat had three spotters equipped with binoculars and a portable mp3 player containing the vocalisations of the birds of Venezuela to identify calls and song. Spot counts were carried out in the morning, when animal activity was greatest. However, due to the logistics of moving camp, there were several occasions when we were required to continue surveys into the afternoon, when activity generally declined. The boat was kept to mid-river when it was narrow enough to cover both banks, but when the river widened to over $40 \mathrm{~m}$ we kept within $15 \mathrm{~m}$ of one bank and a constant speed of approximately $3 \mathrm{~km} / \mathrm{h}$. For birds we used the reference guides of Hilty (2002), Restall et al. (2006) and checklist of Braun et al. (2007), following the nomenclature of Hilty (2002); and for mammals we used Emmons \& Feer (1997). In determining bird species abundance we followed the categorisation of Braun et al. (2007).

\section{Giant otter population estimate}

In surveying the Rewa Head for signs of giant otters we followed the guidelines of the IUCN/SSC Otter Specialist Group
(Groenendijk et al. 2005). The river and its feeding creeks were searched for sign of giant otters during drifts downstream. The position and age of holts, latrines or scratch walls were recorded in order to determine areas of high giant otter activity. These parts of the river were then targeted for observing packs. We used two Canon EOS 400D cameras with $300 \mathrm{~mm}$ and $500 \mathrm{~mm}$ lenses and a Sony Handycam mini DV to capture throat markings allowing individual identification and avoiding double-counting (Hajek et al. 2005). Minimum population estimates were derived from these individual sightings.

\section{Mist netting}

We used three 40ft standard BTO NR nets with a mesh size of $3 \mathrm{~cm}$. The nets were erected 50-200 m from camp in a variety of habitats Nets were erected at 6:00 AM and taken down at 5:30 PM during surveys and were checked every hour. Five netting sites were used over the length of the Rewa Head covering a variety of habitats from open, scrubby bush, to palm thickets and dense forest.

\section{Results}

\section{Mammals}

In total we recorded the presence of 33 species of large mammal, including two marsupials, four xenarthrans, eight primates, ten carnivores, one perrissodactyl, two artiodactyls, and six rodents (Table 1). The camera traps accumulated a total of 5227 hours, resulting in 214 triggering events. Of these, 167 individual animals could be identified from 16 species of mammal (Figure 2). The mammals most frequently observed were the red rumped agouti (Dasyprocta cristata), paca (Agouti paca) and green acouchy (Myoprocta exilis). Four species of felid were observed in the camera traps and jaguar (Panthera onca) were observed from the boat during drift surveys. Lowland tapir (Tapirus terrestris) appeared common above Corona Falls, with nine individuals recorded in the camera traps and four encountered during drift surveys. We found a high diversity of primates during the drift surveys, with all of Guyana's eight species recorded. Of particular importance were the Guiana Shield endemics: the black spider monkey (Ateles paniscus) classified as 'Vulnerable' by the IUCN, the Guianan saki (Pithecia pithecia), and the Guianan red howler monkey (Alouatta macconnelli), recently upgraded to full species. Fresh footprints of bush dog (Speothos venaticus) seen investigating the burrow of a paca along the banks of a tributary feeding the Rewa, and burrows of giant armadillo (Priodontes maximus) are firm evidence for the presence of these threatened species.

\section{Giant otter population size}

We recorded the presence of five different giant otter packs, with a minimum population size of 35 animals. Over the $95 \mathrm{~km}$ surveyed above Corona Falls, this gives a crude density of one otter per $2.7 \mathrm{~km}$ of river. Twenty dens were observed, of which 11 showed signs of recent occupation, and 24 latrines were recorded, of which eight had been used within the last 24 hours.

\section{Birds}

The five netting sites accumulated 420 mist net hours. Eighty-six birds were caught, resulting in 41 different species being identified (Table 2). Twenty three of the species caught in mist nets were not observed during the drift surveys. The most frequently caught family was the Thamnophilidae with 15 of all species and $27 \%$ of total number of individuals caught, followed by the Dendrocolaptidae (5 species) and Trochilidae (4 species). The most common 
Pickles, R.S.A. et al.

Table 1. List of the mammals species recorded in the Rewa Head, showing the form of record, conservation status and level of endemism.

Tabela 1. Lista de mamíferos registradas na cabeceira do Rio Rewa, apresentando a forma de amostragem, status da conservação e endemismos.

\begin{tabular}{|c|c|c|c|}
\hline $\begin{array}{c}\text { Families and } \\
\text { species }\end{array}$ & $\begin{array}{c}\text { Common } \\
\text { name }\end{array}$ & $\begin{array}{r}\text { Conservation } \\
\text { and endemism } \\
\end{array}$ & $\begin{array}{c}\text { ZSL Expedition } 2009 \\
\text { Evidence }\end{array}$ \\
\hline \multicolumn{4}{|l|}{ DIDELPHIAE } \\
\hline Philander opossum & Common Grey Four-eyed Opossum & - & $\mathrm{S}, \mathrm{C}$ \\
\hline Didelphis marsupialis & Common Opossum & - & $\mathrm{C}$ \\
\hline \multicolumn{4}{|l|}{ EDENTATA } \\
\hline $\begin{array}{l}\text { Bradypus tridactylus } \\
\text { MYRMECOPHAGIDAE }\end{array}$ & Pale-throated Three-toed Sloth & GS & $\mathrm{S}$ \\
\hline $\begin{array}{l}\text { Myrmecophaga tridactyla } \\
\text { DASYPODIDAE }\end{array}$ & Giant Anteater & NT & $\mathrm{C}$ \\
\hline Dasypus novemcinctus & Nine-banded Long-nosed Armadillo & - & C,I \\
\hline Priodontes maximus & Giant Armadillo & V & I \\
\hline \multicolumn{4}{|l|}{ CALLITRICHIDAE } \\
\hline Seguinus midas & Golden-handed Tamarin & GS & $\mathrm{S}$ \\
\hline \multicolumn{4}{|l|}{ CEBIDAE } \\
\hline Cebus apella & Brown Capuchin & - & $\mathrm{S}$ \\
\hline Cebus olivaceus & Wedge-capped Capuchin & GS & $\mathrm{S}$ \\
\hline Saimiri sciureus & Common Squirrel Monkey & - & $\mathrm{S}$ \\
\hline Alouatta macconnelli & Guianan Red Howler Monkey & GS & $\mathrm{S}, \mathrm{V}$ \\
\hline Pithecia pithecia & Guianan Saki & GS & $\mathrm{S}$ \\
\hline Chiropotes chiropotes & Red-backed Bearded Saki & GS & $\mathrm{S}$ \\
\hline Ateles paniscus & Black Spider Monkey & GS, V & $\mathrm{S}, \mathrm{V}$ \\
\hline \multicolumn{4}{|l|}{ CANIDAE } \\
\hline Speothos venaticus & Bush Dog & NT & I \\
\hline \multicolumn{4}{|l|}{ PROCYONIDAE } \\
\hline Nasua nasua & South American Coati & - & $\mathrm{S}$ \\
\hline \multicolumn{4}{|l|}{ MUSTELIDAE } \\
\hline Pteronura brasiliensis & Giant Otter & $\mathrm{E}$ & $\mathrm{S}, \mathrm{I}$ \\
\hline Lontra longicaudis & Neotropical Otter & - & $\mathrm{S}, \mathrm{I}$ \\
\hline Eira barbara & Tayra & - & $\mathrm{C}$ \\
\hline \multicolumn{4}{|l|}{ FELIDAE } \\
\hline Panthera onca & Jaguar & NT & $\mathrm{S}, \mathrm{C}, \mathrm{I}$ \\
\hline Puma concolor & Puma & - & $\mathrm{C}$ \\
\hline Leopardus pardalis & Ocelot & - & $\mathrm{C}$ \\
\hline Leopardus wiedii & Margay & NT & $\mathrm{C}$ \\
\hline Felis Jaguarundi & Jaguarundi & - & $\mathrm{S}, \mathrm{C}$ \\
\hline \multicolumn{4}{|l|}{ CERVIDAE } \\
\hline $\begin{array}{l}\text { Mazama americana } \\
\text { TAYASSUIDAE }\end{array}$ & Red Brocket Deer & - & $\mathrm{C}$ \\
\hline Tayassu tajacu & Collared Peccary & - & $\mathrm{S}, \mathrm{C}, \mathrm{I}$ \\
\hline \multicolumn{4}{|l|}{ TAPIRIDAE } \\
\hline $\begin{array}{l}\text { Tapirus terrestris } \\
\text { SCIURIDAE }\end{array}$ & Brazilian Tapir & $\mathrm{V}$ & S,C,I \\
\hline \multicolumn{2}{|l|}{ HYDROCHAERIDAE } & - & $\mathrm{C}$ \\
\hline \multicolumn{3}{|l|}{ Agoutidae } & S,I \\
\hline Agouti paca & Paca & - & S,C,I \\
\hline \multicolumn{4}{|l|}{ DASYPROCTIDAE } \\
\hline Dasyprocta cristata & Red-rumped Agouti & - & $\mathrm{S}, \mathrm{C}, \mathrm{I}$ \\
\hline Myoprocta exilis & Green Acouchy & - & $\mathrm{C}$ \\
\hline ECHIMYIDAE & & & \\
\hline Lonchothrix emiliae & Spiny tree rat & - & $\mathrm{S}$ \\
\hline
\end{tabular}

Evidence of presence is described as S (sighting), V (vocalisation), C (camera trap) or I (indirect evidence) such as footprints, scats or burrows. Conservation status is described as NT (near threatened), V (vulnerable), E (endangered). GS (Guiana Shield) refers to a regional endemic.

A evidência da presença de determinada espécie está descrito como S (avistagens), V (vocalização), C (armadilha fotográfica) ou I (evidência indireta), como pegadas, fezes ou tocas. O status da conservação está descrito como NT (ameaçada), V (vulnerável), E (em perigo). GS (Escudo da Guiana) se refere à região de endemismos. 


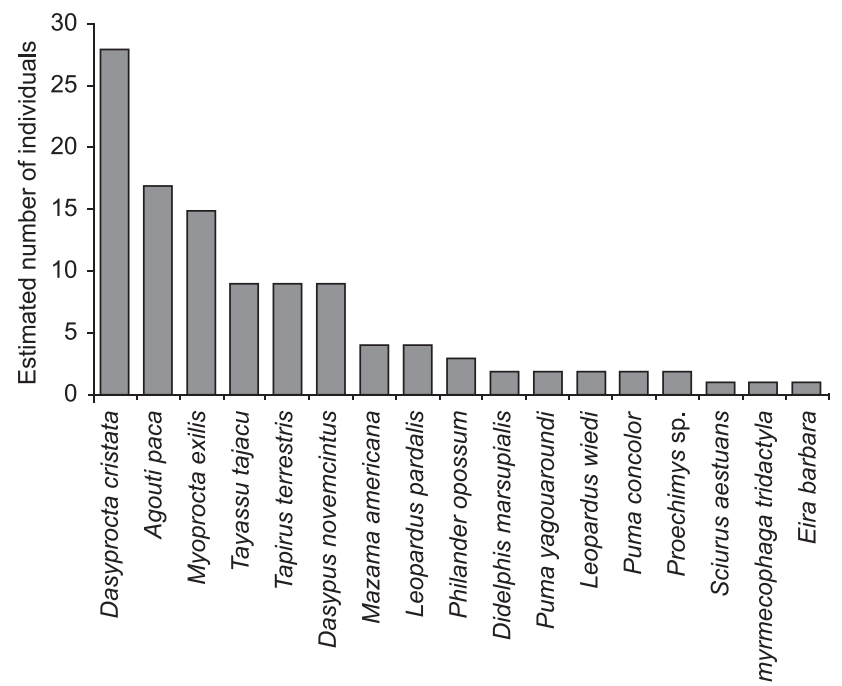

Figure 2. Relative abundance of mammal species recorded in the camera traps.

Figura 2. Abundância relativa de espécies de mamíferos registradas pelas armadilhas fotográficas.

species encountered in the nets was the wedge-billed woodcreeper (Glyphorynchus spirurus), with 11 individuals caught, comprising $13 \%$ of the total number of individuals caught. We recorded over 4000 birds during the drift surveys, resulting in the identification of 158 species through both visual observation and vocalisations. Through the combination of sightings made on drift transects, mist-netting, opportunistic sightings and vocalisations, we positively identified 193 species from 47 different families. The most diverse family observed was the Tyrannidae (34 species) and the most abundant family was the Hirudinidae, comprising $20 \%$ of total observations (Figure 3). Of chief interest in the sightings are 10 Guiana Shield endemics, the Guianan toucanet (Selenidera culik), green aracari (Pteroglossus viridis), black nunbird (Monasa atra), rufousthroated antbird (Gymnopithys rufigula), brown-bellied antwren (Myrmotherula gutturalis), rufous-belled antwren (Myrmotherula guttata), caica parrot (Pionopsitta caica), black curassow (Crax alector), Todd's antwren (Herpsilochmus stictocephalus) and little hermit (Phaethornis longuemareus). Two species recorded are rare throughout their ranges and classified as 'Near Threatned' in the IUCN Red List, the harpy eagle (Harpia harpyja) and crested eagle (Morphnus guianensis).

\section{Discussion}

While far from complete, this expedition revealed a high diversity of bird and mammal species in the Rewa Head of Southern Guyana, including a number of Guiana Shield endemics and the presence of $50 \%$ of Guyana's threatened species (IUCN 2008).

Species accumulation curves (Figure 4) reveal that 96-100\% of species observed in the spot count and camera trap survey were recorded after 12 days, after which time catch per unit effort plateaued. By contrast, the mist net species accumulation curve shows a linear trend. These data suggest that further survey work mist-netting beyond twelve days is required, while a camera trap survey of much longer duration, more traps and covering more habitat types would be necessary to conduct a thorough species inventory.

The high diversity of raptor species is a reflection of a strong prey base, and the presence of both the threatened harpy (Harpia harpyja) and crested eagle (Morphnus guianensis) is likely to be due to both the high cracid, primate and sloth abundance coupled with
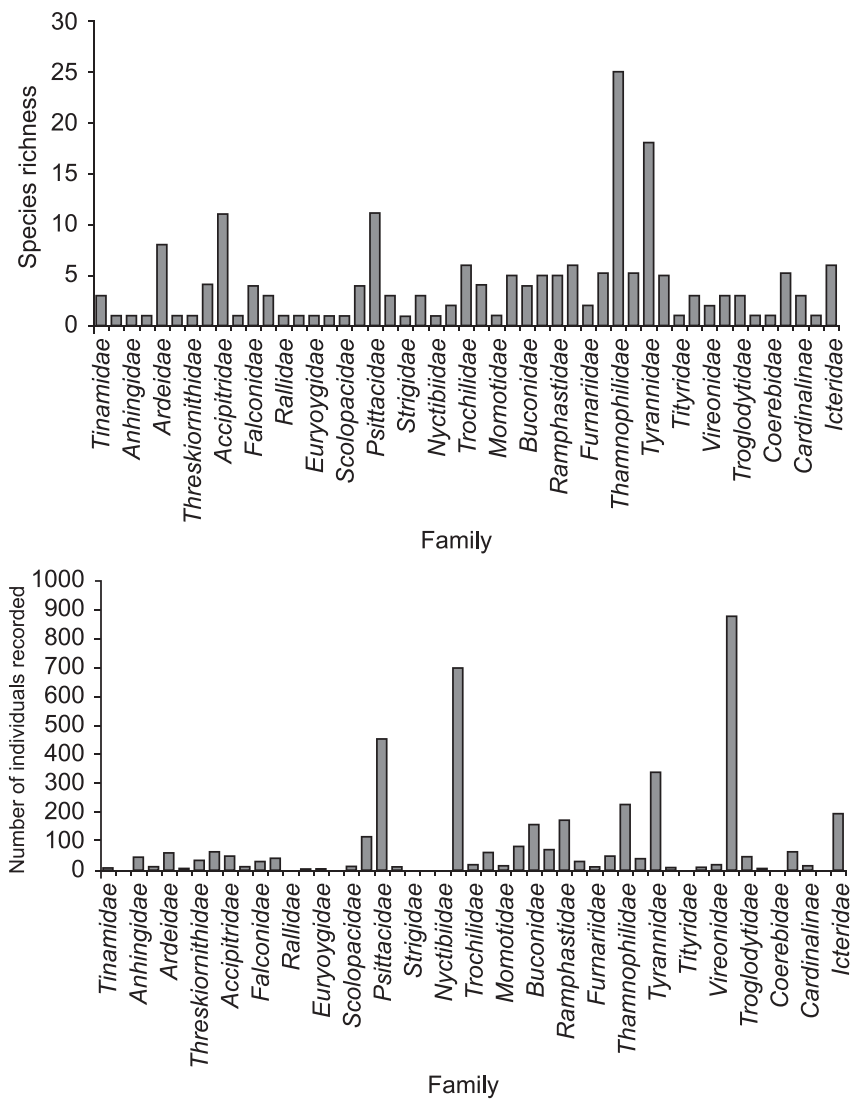

Figure 3. Avifauna species richness and relative abundance by family.

Figura 3. Avifauna riqueza de espécies e abundância relativa por familia.

large mature kapok trees (Ceiba pentandra) providing nesting sites (Touchton et al. 2002).

Comparing the results of our January 2009 expedition with that of a Smithsonian Institution (SI) expedition in August 2006 gives a fuller picture of avian community composition and species richness as well as seasonal variances in local abundance. During the Smithsonian Institution expedition, they set up two mist netting sites above Corona Falls, using 20 nets and netting for 10 days (Milensky \& Schmidt 2006). The combined ZSL/SI figure for bird species in the Rewa Head comes to 250 and number of families to 54 , equating to $30 \%$ of all Guyana's bird species (812). The difference in season between the August 2006 SI expedition and the January 2009 ZSL expedition is most apparent in the presence in January of migratory species such as the Osprey (Pandion haliaetus) and the relative abundance of frugivorous species such as the Psittacidae which were likely more noticeable in January due to congregations forming on fruiting trees. Likewise, whereas the Smithsonian expedition recorded the family Ictericidae as uncommon, we often encountered large flocks of yellow-rumped cacique (Cacicus cela), red-rumped cacique (Cacicus haemorrhous) and crested oropendola (Psarocolius decumanus) as they were nesting, leading us to surmise that they were common in the area.

The 33 species of large mammals we recorded equates to $35 \%$ of Guyana's total non-volant, non-marine mammalian fauna. A typical high prey to low predator encounter rate was recorded, but the presence of five of the Guinan Shield's six species of felid should be noted as important, and there is no reason to believe that the sixth species, the oncilla (Leopardus tigrinus), would not be recorded during the course of a more comprehensive survey. The area appears 
Table 2. List of the bird species recorded in the Rewa Head, showing the abundance, conservation status and level of endemism.

Tabela 2. Lista de espécies de aves registradas nas cabeceiras do Rio Rewa, apresentando abundância, status da conservação e endemismos.

\begin{tabular}{|c|c|c|c|c|c|c|}
\hline \multirow{2}{*}{$\begin{array}{l}\text { Families and } \\
\text { species }\end{array}$} & \multirow{2}{*}{$\begin{array}{l}\text { Common } \\
\text { name }\end{array}$} & \multirow{2}{*}{$\begin{array}{c}\text { Conservation } \\
\text { and } \\
\text { endemism }\end{array}$} & \multirow{2}{*}{$\begin{array}{c}\text { SI } \\
\text { expedition } \\
\text { Aug 2006 } \\
\text { abundance }\end{array}$} & \multicolumn{3}{|c|}{ ZSL expedition Jan./2009 } \\
\hline & & & & Abundance & $\begin{array}{c}\text { Recorded } \\
\text { during } \\
\text { river counts }\end{array}$ & $\begin{array}{c}\text { Caught } \\
\text { in mist } \\
\text { nets }\end{array}$ \\
\hline \multicolumn{7}{|l|}{ TINAMIDAE } \\
\hline Tinamus major & Great Tinamou & - & $\mathrm{F}$ & S & 2 & - \\
\hline Crypturellus cinereus & Cinereous Tinamou & - & S & $S$ & 4 & - \\
\hline Crypturellus variegatus & Variegated Tinamou & - & $\mathrm{F}$ & $S$ & $*$ & - \\
\hline ANATIDAE & & & & & & \\
\hline $\begin{array}{l}\text { Cairina moschata } \\
\text { ANHINGIDAE }\end{array}$ & Muscovy duck & - & - & $S$ & $*$ & - \\
\hline $\begin{array}{l}\text { Anhinga anhinga } \\
\text { PHALACROCORACIDAE }\end{array}$ & Anhinga & - & $\mathrm{F}$ & $\mathrm{F}$ & 43 & - \\
\hline $\begin{array}{l}\text { Phalacrocorax brasiliensis } \\
\text { ARDEIDAE }\end{array}$ & Neotropical Cormorant & - & S & S & 6 & - \\
\hline Zebrilus undualtus & Zigzag Heron & - & S & - & - & - \\
\hline Tigrisoma lineatum & Rufescent Tiger-Heron & - & $\mathrm{U}$ & $\mathrm{U}$ & 8 & - \\
\hline Tigrisoma fasciatum & Fasciated Tiger-Heron & - & $\mathrm{U}$ & $S$ & 1 & - \\
\hline Ardea cocoi & Cocoi Heron & - & $\mathrm{F}$ & $\mathrm{F}$ & 44 & - \\
\hline Egretta caerulea & Little Blue Heron & - & & S & $*$ & - \\
\hline Bubulcus ibis & Cattle Egret & - & $\mathrm{S}$ & - & - & - \\
\hline Aredea alba & Great Egret & - & - & S & 2 & - \\
\hline Agamia agami & Agami Heron & - & - & S & 1 & - \\
\hline Butorides striatus & Striated Heron & - & $\mathrm{U}$ & $\mathrm{U}$ & 5 & - \\
\hline $\begin{array}{l}\text { Pilherodias pileatus } \\
\text { CICONIIDAE }\end{array}$ & Capped Heron & - & $\mathrm{U}$ & S & $*$ & - \\
\hline $\begin{array}{r}\text { Mycteria americana } \\
\text { THRESKIORNITHIDAE }\end{array}$ & Wood Stork & - & - & S & 2 & - \\
\hline $\begin{array}{l}\text { Mesembrinibis cayennensis } \\
\text { CATHARTIDAE }\end{array}$ & Green Ibis & - & $\mathrm{C}$ & F & 32 & - \\
\hline Coragyps atratus & Black Vulture & - & $\mathrm{U}$ & $\mathrm{U}$ & 6 & - \\
\hline Cathartes aura & Turkey Vulture & - & & $S$ & 1 & - \\
\hline Cathartes melanbrotus & $\begin{array}{c}\text { Greater Yellow-headed } \\
\text { Vulture }\end{array}$ & - & $\mathrm{F}$ & $\mathrm{F}$ & 34 & - \\
\hline $\begin{array}{l}\text { Sarcoramphus papa } \\
\text { ACCIPITRIDAE }\end{array}$ & King Vulture & - & $\mathrm{F}$ & $\mathrm{F}$ & 23 & - \\
\hline Elanoides forficatus & Swallow-tailed Kite & - & S & $\mathrm{F}$ & 17 & - \\
\hline Harpagus bidentatus & Double-toothed Kite & - & $S$ & & & - \\
\hline Leptodon cayanensis & Grey-headed kite & - & - & S & 2 & - \\
\hline Ictinia plumbea & Plumbeous Kite & - & S & S & 1 & - \\
\hline Buteogallus urubitinga & Great Black-Hawk & - & $\mathrm{U}$ & $\mathrm{U}$ & 12 & - \\
\hline Leucopternis albicollis & White Hawk & - & - & $\mathrm{U}$ & 8 & - \\
\hline Buteo magnirostris & Roadside Hawk & - & $\mathrm{U}$ & S & $*$ & - \\
\hline Buteo brachyurus & Short-tailed Hawk & - & - & S & 2 & - \\
\hline Accipiter superciliosus & Tiny Hawk & - & - & S & 1 & - \\
\hline Harpia harpyja & Harpy Eagle & NT & - & S & $*$ & - \\
\hline Morphnus guianensis & Crested Eagle & NT & - & S & 1 & - \\
\hline $\begin{array}{l}\text { Spizaetus ornatus } \\
\text { PANDIONIDAE }\end{array}$ & Ornate Hawk Eagle & - & - & $S$ & 3 & - \\
\hline
\end{tabular}

Conservation status is described as NT (near threatened), V (vulnerable), E (endangered). GS (Guiana Shield) refers to a regional endemic. Abundance is recorded as $\mathrm{C}$ (common), more than 20 individuals encountered daily in prime habitat and season; $\mathrm{F}$ (fairly common) 5-20 individuals encountered daily in prime habitat and season; $\mathrm{U}$ (uncommon), fewer than 5 individuals encountered, not encountered daily even in prime habitat and season; $\mathrm{S}$ (scarce) only occasionally encountered in small numbers even in prime habitat and season; * Recorded present above Corona Falls, but not recorded during a survey.

O status da conservação está descrito como NT (ameaçada), V (vulnerável), E (em perigo). GS (Escudo da Guiana) se refere à região de endemismos. A abundância foi registrada como C (comum), quando mais de 20 indivíduos foram encontrados diariamente em seu habitat original e estação ótimos; F (relativamente comum) 5-20 indivíduos encontrados diariamente em seu habitat e estação ótimos; U (incomum) menos de 5 indivíduos encontrados; não encontrados diariamente mesmo no habitat original e estação ótimos; S (raro) apenas ocasionalmente encontrados em pequeno número mesmo no habitat original e estação ótimos. * Indivíduos encontrados acima Corona Falls, mas não encontrados durante um exame. 
Table 2. Continued...

Tabela 2. Continuação...

\begin{tabular}{|c|c|c|c|c|c|c|}
\hline \multirow{2}{*}{$\begin{array}{l}\text { Families and } \\
\text { species }\end{array}$} & \multirow{2}{*}{$\begin{array}{l}\text { Common } \\
\text { name }\end{array}$} & \multirow{2}{*}{$\begin{array}{c}\text { Conservation } \\
\text { and } \\
\text { endemism }\end{array}$} & \multirow{2}{*}{$\begin{array}{c}\text { SI } \\
\text { expedition } \\
\text { Aug } 2006 \\
\text { abundance } \\
\end{array}$} & \multicolumn{3}{|c|}{ ZSL expedition Jan./2009 } \\
\hline & & & & Abundance & $\begin{array}{l}\text { Recorded } \\
\text { during } \\
\text { river counts }\end{array}$ & $\begin{array}{c}\text { Caught } \\
\text { in mist } \\
\text { nets }\end{array}$ \\
\hline $\begin{array}{l}\text { Pandion haliaetus } \\
\text { FALCONIDAE }\end{array}$ & Osprey & - & - & $S$ & 7 & - \\
\hline Micrastur gilvicollis & Lined Forest-Falcon & - & $\mathrm{U}$ & - & - & - \\
\hline Micrastur semitorquatus & Collared Forest-Falcon & - & - & S & 1 & - \\
\hline Daptrius ater & Black Caracara & - & $\mathrm{U}$ & $\mathrm{U}$ & 10 & - \\
\hline Ibycter americanus & Red-throated Caracara & - & $\mathrm{F}$ & $\mathrm{U}$ & 14 & - \\
\hline Falco rufigularis & Bat Falcon & - & $\mathrm{F}$ & $\mathrm{U}$ & 3 & - \\
\hline CRACIDAE & & & & & & \\
\hline Ortalis motmot & Little Chachalaca & - & $\mathrm{U}$ & $\mathrm{U}$ & 9 & - \\
\hline Crax alector & Black Curassow & GS & $\mathrm{U}$ & $\mathrm{F}$ & 19 & - \\
\hline $\begin{array}{r}\text { Pipile cumanensis } \\
\text { ODONTOPHORIDAE }\end{array}$ & Blue-throated Piping-Guan & - & $\mathrm{F}$ & $\mathrm{U}$ & 12 & - \\
\hline $\begin{array}{l}\text { Odontophorus gujanensis } \\
\text { RALLIDAE }\end{array}$ & Marbled Wood-Quail & - & $\mathrm{U}$ & - & - & - \\
\hline $\begin{array}{l}\text { Aramides cajanea } \\
\text { HELIORNITHIDAE }\end{array}$ & Grey-necked Wood-Rail & - & $\mathrm{S}$ & S & 1 & - \\
\hline $\begin{array}{l}\text { Heliornis fulica } \\
\text { EURYPYGIDAE }\end{array}$ & Sungrebe & - & $\mathrm{U}$ & S & 3 & - \\
\hline $\begin{array}{l}\text { Eurypyga helias } \\
\text { PSOPHIDAE }\end{array}$ & Sunbittern & - & $\mathrm{U}$ & $S$ & 3 & - \\
\hline $\begin{array}{l}\text { Psophia crepitans } \\
\text { SCOLOPACIDAE }\end{array}$ & Grey-winged Trumpeter & - & $\mathrm{U}$ & $S$ & $*$ & - \\
\hline $\begin{array}{l}\text { Actitis macularia } \\
\text { COLUMBIDAE }\end{array}$ & Spotted Sandpiper & - & $\mathrm{F}$ & $\mathrm{U}$ & 13 & - \\
\hline Columba plumbea & Plumbeous Pigeon & - & $\mathrm{C}$ & F & 30 & - \\
\hline Columba subvinacea & Ruddy Pigeon & - & $\mathrm{C}$ & $\mathrm{F}$ & 30 & - \\
\hline Leptotila rufaxilla & Grey-fronted Dove & - & $\mathrm{U}$ & $\mathrm{F}$ & 55 & 2 \\
\hline $\begin{array}{l}\text { Geotrygon montana } \\
\text { PSITTACIDAE }\end{array}$ & Ruddy Quail-Dove & - & $\mathrm{F}$ & & & - \\
\hline Pyrrhura picta & Painted Parakeet & - & $\mathrm{C}$ & $\mathrm{C}$ & 46 & - \\
\hline Brotogeris chrysoptera & Golden-winged Parakeet & - & & S & $*$ & - \\
\hline Ara ararauna & Blue-and-Gold Macaw & - & $\mathrm{U}$ & $\mathrm{F}$ & 31 & - \\
\hline Ara chloropterus & Red-and-green Macaw & - & $\mathrm{F}$ & $\mathrm{F}$ & 21 & - \\
\hline Ara macao & Scarlet Macaw & - & $\mathrm{U}$ & F & 51 & - \\
\hline Brotogeris chrysopterus & Golden-winged Parakeet & - & $\mathrm{C}$ & $\mathrm{C}$ & 73 & - \\
\hline Pionus menstruus & Blue-headed parrot & - & & $\mathrm{C}$ & 96 & - \\
\hline Pionopsitta caica & Caica Parrot & GS & $\mathrm{C}$ & $\mathrm{U}$ & 17 & - \\
\hline Amazona amazonica & Orange-winged Parrot & - & $\mathrm{U}$ & $\mathrm{C}$ & 98 & - \\
\hline Amazona ochrocephala & Yellow-crowned Parrot & - & $\mathrm{U}$ & & & - \\
\hline Amazona farinosa & Mealy Parrot & - & & $\mathrm{U}$ & 10 & - \\
\hline $\begin{array}{l}\text { Deroptyus accipitrinus } \\
\text { CUCULIDAE }\end{array}$ & Red-fan Parrot & - & $\mathrm{F}$ & $\mathrm{U}$ & 10 & - \\
\hline Piaya cayana & Squirrel Cuckoo & - & $\mathrm{F}$ & $\mathrm{U}$ & 12 & - \\
\hline Piaya minuta & Little Cuckoo & - & - & S & 1 & - \\
\hline Crotophaga major & Greater Ani & - & $\mathrm{U}$ & $\mathrm{S}$ & $*$ & - \\
\hline
\end{tabular}

Conservation status is described as NT (near threatened), V (vulnerable), E (endangered). GS (Guiana Shield) refers to a regional endemic. Abundance is recorded as C (common), more than 20 individuals encountered daily in prime habitat and season; F (fairly common) 5-20 individuals encountered daily in prime habitat and season; $\mathrm{U}$ (uncommon), fewer than 5 individuals encountered, not encountered daily even in prime habitat and season; $\mathrm{S}$ (scarce) only occasionally encountered in small numbers even in prime habitat and season; * Recorded present above Corona Falls, but not recorded during a survey.

O status da conservação está descrito como NT (ameaçada), V (vulnerável), E (em perigo). GS (Escudo da Guiana) se refere à região de endemismos. A abundância foi registrada como C (comum), quando mais de 20 indivíduos foram encontrados diariamente em seu habitat original e estação ótimos; F (relativamente comum) 5-20 indivíduos encontrados diariamente em seu habitat e estação ótimos; U (incomum) menos de 5 indivíduos encontrados; não encontrados diariamente mesmo no habitat original e estação ótimos; S (raro) apenas ocasionalmente encontrados em pequeno número mesmo no habitat original e estação ótimos. * Indivíduos encontrados acima Corona Falls, mas não encontrados durante um exame. 
Table 2. Continued...

Tabela 2. Continuação...

\begin{tabular}{|c|c|c|c|c|c|c|}
\hline \multirow{2}{*}{$\begin{array}{l}\text { Families and } \\
\text { species }\end{array}$} & \multirow{2}{*}{$\begin{array}{c}\text { Common } \\
\text { name }\end{array}$} & \multirow{2}{*}{$\begin{array}{c}\text { Conservation } \\
\text { and } \\
\text { endemism }\end{array}$} & \multirow{2}{*}{$\begin{array}{c}\text { SI } \\
\text { expedition } \\
\text { Aug } 2006 \\
\text { abundance }\end{array}$} & \multicolumn{3}{|c|}{ ZSL expedition Jan./2009 } \\
\hline & & & & Abundance & $\begin{array}{l}\text { Recorded } \\
\text { during } \\
\text { river counts }\end{array}$ & $\begin{array}{c}\text { Caught } \\
\text { in mist } \\
\text { nets }\end{array}$ \\
\hline \multicolumn{7}{|l|}{ STRIGIDAE } \\
\hline Otus watsoni & Tawny-bellied Screech-Owl & - & $\mathrm{U}$ & - & - & - \\
\hline Pulsatrix perspicillata & Spectacled Owl & - & $\mathrm{U}$ & S & 1 & - \\
\hline Glaucidium hardyii & Amazonian Pygmy-Owl & - & $\mathrm{F}$ & & & - \\
\hline \multicolumn{7}{|l|}{ CAPRIMULGIDAE } \\
\hline Lurocalis semitorquatus & Short-tailed Nighthawk & - & $\mathrm{U}$ & $\mathrm{U}$ & $*$ & - \\
\hline Nyctidromus albicollis & Common Pauraque & - & - & $\mathrm{F}$ & * & - \\
\hline Caprimulgus nigrescens & Blackish Nightjar & - & $\mathrm{U}$ & $\mathrm{U}$ & 2 & 2 \\
\hline \multicolumn{7}{|l|}{ NYCTIBIIDAE } \\
\hline \multicolumn{7}{|l|}{ APODIDAE } \\
\hline Chaetura spinicauda & Band-rumped Swift & - & $\mathrm{C}$ & $\mathrm{C}$ & 707 & - \\
\hline Chaetura cinereiventris & Grey-rumped Swift & - & - & $\mathrm{S}$ & $*$ & - \\
\hline \multicolumn{7}{|l|}{ TROCHILIDAE } \\
\hline Phaethornis superciliosus & Eastern Long-tailed Hermit & - & $\mathrm{U}$ & $\mathrm{U}$ & $*$ & 7 \\
\hline Phaethornis bourcieri & Straight-billed Hermit & - & $\mathrm{U}$ & - & - & - \\
\hline Phaethornis ruber & Reddish Hermit & - & $\mathrm{F}$ & - & - & - \\
\hline Phaethornis longuemareus & Little hermit & GS & & S & 2 & 1 \\
\hline Campylopterus largipennis & Grey-breasted Sabrewing & - & S & S & - & 3 \\
\hline Florisuga mellivora & White-necked Jacobin & - & $\mathrm{U}$ & - & - & - \\
\hline Thalurania furcata & Fork-tailed Woodnymph & - & $\mathrm{U}$ & S & & 2 \\
\hline Topaza pella & Crimson Topaz & - & $\mathrm{S}$ & S & 3 & - \\
\hline Heliothryx aurita & Black-eared Fairy & - & $\mathrm{S}$ & S & * & - \\
\hline \multicolumn{7}{|l|}{ TROGONIDAE } \\
\hline Trogon viridis & White-tailed Trogon & - & $\mathrm{F}$ & $\mathrm{F}$ & 33 & - \\
\hline Trogon violaceus & Violaceous Trogon & - & $\mathrm{U}$ & $\mathrm{U}$ & 24 & - \\
\hline Trogon collaris & Collared Trogon & - & $\mathrm{F}$ & - & - & - \\
\hline Trogon personatus & Masked Trogon & - & $\mathrm{U}$ & - & - & - \\
\hline Trogon melanurus & Black-tailed Trogon & - & - & S & 2 & - \\
\hline \multicolumn{7}{|l|}{ MOMOTIDAE } \\
\hline Momotus momota & Blue-crowned Motmot & - & $\mathrm{F}$ & $\mathrm{U}$ & 14 & 1 \\
\hline \multicolumn{7}{|l|}{ ALCEDINIDAE } \\
\hline Ceryle torquata & Ringed Kingfisher & - & $\mathrm{C}$ & $\mathrm{U}$ & 19 & - \\
\hline Chloroceryle amazona & Amazon Kingfisher & - & $\mathrm{C}$ & $\mathrm{F}$ & 33 & 1 \\
\hline Chloroceryle americana & Green Kingfisher & - & $\mathrm{U}$ & $\mathrm{F}$ & 24 & - \\
\hline Chloroceryle inda & $\begin{array}{l}\text { Green-and-Rufous } \\
\text { Kingfisher }\end{array}$ & - & $\mathrm{U}$ & $\mathrm{S}$ & 3 & \\
\hline $\begin{array}{l}\text { Chloroceryle aenea } \\
\text { BUCCONIDAE }\end{array}$ & Pygmy Kingfisher & - & $\mathrm{S}$ & S & 1 & 1 \\
\hline Notharchus macrorhyncus & Guianan puffbird & - & - & S & 2 & - \\
\hline Bucco capensis & Collared Puffbird & - & $\mathrm{U}$ & S & 3 & - \\
\hline Malacoptila fusca & White-chested Puffbird & - & $\mathrm{U}$ & - & - & - \\
\hline Monasa atra & Black Nunbird & GS & $\mathrm{F}$ & $\mathrm{F}$ & 37 & - \\
\hline $\begin{array}{l}\text { Chelidoptera tenebrosa } \\
\text { GALBULIDAE }\end{array}$ & Swallow-wing Puffbird & - & $\mathrm{C}$ & $\mathrm{C}$ & 113 & - \\
\hline
\end{tabular}

Conservation status is described as NT (near threatened), V (vulnerable), E (endangered). GS (Guiana Shield) refers to a regional endemic. Abundance is recorded as $\mathrm{C}$ (common), more than 20 individuals encountered daily in prime habitat and season; F (fairly common) 5-20 individuals encountered daily in prime habitat and season; U (uncommon), fewer than 5 individuals encountered, not encountered daily even in prime habitat and season; S (scarce) only occasionally encountered in small numbers even in prime habitat and season; * Recorded present above Corona Falls, but not recorded during a survey.

O status da conservação está descrito como NT (ameaçada), V (vulnerável), E (em perigo). GS (Escudo da Guiana) se refere à região de endemismos. A abundância foi registrada como C (comum), quando mais de 20 indivíduos foram encontrados diariamente em seu habitat original e estação ótimos; F (relativamente comum) 5-20 indivíduos encontrados diariamente em seu habitat e estação ótimos; U (incomum) menos de 5 indivíduos encontrados; não encontrados diariamente mesmo no habitat original e estação ótimos; S (raro) apenas ocasionalmente encontrados em pequeno número mesmo no habitat original e estação ótimos. * Indivíduos encontrados acima Corona Falls, mas não encontrados durante um exame. 
Table 2. Continued...

Tabela 2. Continuação...

\begin{tabular}{|c|c|c|c|c|c|c|}
\hline \multirow{2}{*}{$\begin{array}{l}\text { Families and } \\
\text { species }\end{array}$} & \multirow{2}{*}{$\begin{array}{l}\text { Common } \\
\text { name }\end{array}$} & \multirow{2}{*}{$\begin{array}{c}\text { Conservation } \\
\text { and } \\
\text { endemism }\end{array}$} & \multirow{2}{*}{$\begin{array}{c}\text { SI } \\
\text { expedition } \\
\text { Aug 2006 } \\
\text { abundance }\end{array}$} & \multicolumn{3}{|c|}{ ZSL expedition Jan./2009 } \\
\hline & & & & Abundance & $\begin{array}{c}\text { Recorded } \\
\text { during } \\
\text { river counts }\end{array}$ & $\begin{array}{c}\text { Caught } \\
\text { in mist } \\
\text { nets }\end{array}$ \\
\hline Galbula galbula & Green-tailed Jacamar & - & $\mathrm{U}$ & $\mathrm{F}$ & 32 & - \\
\hline Galbula albirostris & Yellow-billed Jacamar & - & $\mathrm{F}$ & S & - & 1 \\
\hline Galbula dea & Paradise Jacamar & - & $\mathrm{U}$ & S & 3 & - \\
\hline Jacamerops aurea & Great Jacamar & - & $\mathrm{U}$ & $S$ & 3 & 1 \\
\hline Brachygalba lugubris & Brown jacamar & - & - & $\mathrm{F}$ & 29 & - \\
\hline \multicolumn{7}{|l|}{ RAMPHASTIDAE } \\
\hline Pteroglossus viridis & Green Aracari & GS & - & $\mathrm{U}$ & 12 & - \\
\hline Pteroglossus aracari & Black-necked Aracari & - & $\mathrm{U}$ & S & $*$ & - \\
\hline Selenidera culik & Guianan Toucanet & GS & $\mathrm{U}$ & S & 7 & - \\
\hline Ramphastos vitellinus & Channel-billed Toucan & - & $\mathrm{C}$ & $\mathrm{F}$ & 38 & - \\
\hline Ramphastos tucanus & White-throated Toucan & - & $\mathrm{C}$ & $\mathrm{C}$ & 114 & - \\
\hline \multicolumn{7}{|l|}{ PICIDAE } \\
\hline Dryocopus lineatus & Lineated Woodpecker & - & $S$ & $S$ & 13 & - \\
\hline Veniliornis cassini & $\begin{array}{l}\text { Golden-collared } \\
\text { Woodpecker }\end{array}$ & - & $\mathrm{U}$ & & & \\
\hline Piculus flavigula & $\begin{array}{l}\text { Yellow-throated } \\
\text { Woodpecker }\end{array}$ & - & $\mathrm{F}$ & S & $*$ & - \\
\hline Celeus elegans & Chestnut Woodpecker & - & $\mathrm{F}$ & S & 2 & - \\
\hline Celeus undatus & Waved Woodpecker & - & $\mathrm{S}$ & S & 2 & - \\
\hline Celeus flavus & Cream-colored Woodpecker & - & $\mathrm{U}$ & - & - & - \\
\hline Campephilus rubricollis & Red-necked Woodpecker & - & $\mathrm{F}$ & $S$ & 1 & 1 \\
\hline Campephilus melanoleucos & $\begin{array}{l}\text { Crimson-crested } \\
\text { Woodpecker }\end{array}$ & - & $\mathrm{F}$ & $\mathrm{U}$ & 9 & - \\
\hline \multicolumn{7}{|l|}{ FURNARIIDAE } \\
\hline Philydor pyrrhodes & $\begin{array}{l}\text { Cinnamon-rumped } \\
\text { Foliage-gleaner }\end{array}$ & - & $S$ & - & - & - \\
\hline Philydor erythrocercus & $\begin{array}{l}\text { Rufous-rumped } \\
\text { Foliage-gleaner }\end{array}$ & - & $\mathrm{F}$ & - & - & - \\
\hline Automolus ochrolaemus & $\begin{array}{l}\text { Buff-throated } \\
\text { Foliage-gleaner }\end{array}$ & - & $\mathrm{F}$ & S & - & 1 \\
\hline Automolus rufipileatus & $\begin{array}{l}\text { Chestnut-crowned } \\
\text { Foliage-gleaner }\end{array}$ & - & - & $\mathrm{U}$ & 12 & \\
\hline $\begin{array}{c}\text { Xenops minutus } \\
\text { DENDROCOLAPTIDAE }\end{array}$ & \multicolumn{5}{|c|}{ DENDROCOLAPTIDAE } & - \\
\hline Dendrocincla fuliginosa & Plain-brown Woodcreeper & - & $\mathrm{F}$ & S & 1 & 3 \\
\hline Glyphorynchus spirurus & Wedge-billed Woodcreeper & - & $\mathrm{C}$ & $\mathrm{F}$ & 3 & 11 \\
\hline Dendrocolaptes certhia & $\begin{array}{l}\text { Amazonian Barred } \\
\text { Woodcreeper }\end{array}$ & - & $\mathrm{S}$ & S & 3 & - \\
\hline $\begin{array}{l}\text { Xiphocolaptes } \\
\text { promeropirhynchus }\end{array}$ & Strong-billed Woodcreeper & - & S & S & - & 1 \\
\hline Xiphorhynchus pardalotus & $\begin{array}{l}\text { Chestnut-rumped } \\
\text { Woodcreeper }\end{array}$ & - & $\mathrm{C}$ & - & - & - \\
\hline Xiphorhynchus guttatus & Buff-throated Woodcreeper & - & $\mathrm{U}$ & S & 24 & 2 \\
\hline $\begin{array}{l}\text { Campylorhamphus procurvoides } \\
\text { THAMNOPHILIDAE }\end{array}$ & Curve-billed Scythebill & - & $\mathrm{U}$ & & & \\
\hline Cymbilaimus lineatus & Fasciated Antshrike & - & $\mathrm{F}$ & $\mathrm{S}$ & 7 & - \\
\hline
\end{tabular}

Conservation status is described as NT (near threatened), V (vulnerable), E (endangered). GS (Guiana Shield) refers to a regional endemic. Abundance is recorded as $\mathrm{C}$ (common), more than 20 individuals encountered daily in prime habitat and season; F (fairly common) 5-20 individuals encountered daily in prime habitat and season; $\mathrm{U}$ (uncommon), fewer than 5 individuals encountered, not encountered daily even in prime habitat and season; $\mathrm{S}$ (scarce) only occasionally encountered in small numbers even in prime habitat and season; * Recorded present above Corona Falls, but not recorded during a survey.

O status da conservação está descrito como NT (ameaçada), V (vulnerável), E (em perigo). GS (Escudo da Guiana) se refere à região de endemismos. A abundância foi registrada como C (comum), quando mais de 20 indivíduos foram encontrados diariamente em seu habitat original e estação ótimos; F (relativamente comum) 5-20 indivíduos encontrados diariamente em seu habitat e estação ótimos; U (incomum) menos de 5 indivíduos encontrados; não encontrados diariamente mesmo no habitat original e estação ótimos; S (raro) apenas ocasionalmente encontrados em pequeno número mesmo no habitat original e estação ótimos. * Indivíduos encontrados acima Corona Falls, mas não encontrados durante um exame. 
Table 2. Continued...

Tabela 2. Continuação...

\begin{tabular}{|c|c|c|c|c|c|c|}
\hline \multirow{2}{*}{$\begin{array}{c}\text { Families and } \\
\text { species }\end{array}$} & \multirow{2}{*}{$\begin{array}{c}\text { Common } \\
\text { name }\end{array}$} & \multirow{2}{*}{$\begin{array}{c}\text { Conservation } \\
\text { and } \\
\text { endemism }\end{array}$} & \multirow{2}{*}{$\begin{array}{c}\text { SI } \\
\text { expedition } \\
\text { Aug 2006 } \\
\text { abundance }\end{array}$} & \multicolumn{3}{|c|}{ ZSL expedition Jan./2009 } \\
\hline & & & & Abundance & $\begin{array}{c}\text { Recorded } \\
\text { during } \\
\text { river counts }\end{array}$ & $\begin{array}{c}\text { Caught } \\
\text { in mist } \\
\text { nets } \\
\end{array}$ \\
\hline Frederikena viridis & Black-throated Antshrike & - & $\mathrm{U}$ & - & - & - \\
\hline Taraba major & Great Antshrike & - & & $\mathrm{S}$ & 1 & - \\
\hline Thamnophilus murinus & Mouse-colored Antshrike & - & $\mathrm{F}$ & $\mathrm{U}$ & 5 & - \\
\hline Thamnophilus punctatus & Guianan Slaty-Antshrike & - & $\mathrm{U}$ & - & - & - \\
\hline Pygiptila stellaris & Spot-winged Antshrike & - & $\mathrm{U}$ & - & - & - \\
\hline Thamnomanes ardesiacus & Dusky-throated Antshrike & - & $\mathrm{F}$ & $\mathrm{S}$ & - & 2 \\
\hline Thamnomanes caesius & Cinereous Antshrike & - & $\mathrm{F}$ & $\mathrm{S}$ & 2 & 1 \\
\hline Myrmotherula brachyura & Pygmy Antwren & - & $\mathrm{F}$ & $\mathrm{S}$ & 3 & - \\
\hline Myrmotherula surinamensis & Guianan Streaked Antwren & - & $\mathrm{C}$ & $\mathrm{C}$ & 100 & 1 \\
\hline Myrmotherula guttata & Rufous-bellied Antwren & GS & $\mathrm{U}$ & $\mathrm{U}$ & $*$ & 5 \\
\hline Myrmotherula gutturalis & Brown-bellied Antwren & GS & $\mathrm{U}$ & $\mathrm{S}$ & - & 1 \\
\hline Myrmotherula menetriesii & Grey Antwren & - & - & $\mathrm{S}$ & - & 1 \\
\hline Myrmotherula longipennis & Long-winged Antwren & - & $\mathrm{C}$ & $\mathrm{U}$ & - & 2 \\
\hline Herpsilochmus sticturus & Spot-tailed Antwren & - & $\mathrm{F}$ & $\mathrm{F}$ & 14 & - \\
\hline Herpsilochmus stictocephalus & Todd's Antwren & GS & $\mathrm{F}$ & $\mathrm{U}$ & 8 & - \\
\hline Microrhopias quixensis & Dot-winged Antwren & - & $\mathrm{S}$ & - & - & - \\
\hline Terenura spodioptila & Ash-winged Antwren & - & $\mathrm{S}$ & - & - & - \\
\hline Cercomacra cinerascens & Grey Antbird & - & $\mathrm{C}$ & $\mathrm{U}$ & 9 & - \\
\hline Cercomacra tyrannina & Dusky Antbird & - & $\mathrm{F}$ & $\mathrm{U}$ & 9 & 1 \\
\hline Myrmoborus myotherinus & White-browed Antbird & - & - & $\mathrm{U}$ & 7 & - \\
\hline Hypocnemis cantator & Warbling Antbird & - & $\mathrm{F}$ & $\mathrm{F}$ & 14 & 1 \\
\hline Hypocnemoides melanopogon & Black-chinned Antbird & - & $\mathrm{F}$ & $\mathrm{U}$ & 9 & - \\
\hline Percnostola rufifrons & Black-headed Antbird & - & $\mathrm{F}$ & $\mathrm{U}$ & 12 & - \\
\hline Hylophylax poecilinota & Scale-backed Antbird & - & $\mathrm{F}$ & $\mathrm{S}$ & - & 2 \\
\hline Hylophylax naevia & Spot-backed Antbird & - & $\mathrm{F}$ & $\mathrm{S}$ & 1 & 1 \\
\hline Myrmeciza ferruginea & Ferruginous-backed Antbird & - & - & $\mathrm{S}$ & 2 & - \\
\hline Myrmornis torquata & Wing-banded Antbird & - & $\mathrm{U}$ & $\mathrm{S}$ & - & - \\
\hline Pithys albifrons & White-plumed Antbird & - & $\mathrm{F}$ & $\mathrm{S}$ & - & 2 \\
\hline Gymnopithys rufigula & Rufous-throated Antbird & GS & $\mathrm{F}$ & $\mathrm{S}$ & - & 1 \\
\hline Sclateria naevia & Silvered Antbird & - & - & $\mathrm{S}$ & - & 1 \\
\hline FORMICARIIDAE & & & & & & \\
\hline Formicarius analis & Black-faced Antthrush & - & $\mathrm{U}$ & $\mathrm{U}$ & 5 & - \\
\hline Formicarius colma & Rufous-capped Antthrush & - & - & $\mathrm{S}$ & $*$ & 2 \\
\hline Hylopezus macularius & Spotted Antpitta & - & $\mathrm{F}$ & $\mathrm{U}$ & 5 & 1 \\
\hline Myrmothera campanisona & Thrush-like Antpitta & - & $\mathrm{F}$ & $\mathrm{F}$ & 21 & - \\
\hline Grallaria varia & Variegated Antpitta & - & - & $\mathrm{S}$ & 1 & 1 \\
\hline CONOPOPHAGIDAE & & & & & & \\
\hline Conopophaga aurita & Chestnut-belted Gnateater & - & $\mathrm{U}$ & - & - & - \\
\hline TYRANNIDAE & & & & & & \\
\hline Tyrannulus elatus & Yellow-crowned Tyrannulet & - & $\mathrm{F}$ & - & - & - \\
\hline Myiopagis gaimardii & Forest Elaenia & - & $\mathrm{U}$ & $\mathrm{U}$ & 11 & - \\
\hline Mionectes macconnelli & McConnell's Flycatcher & - & $\mathrm{U}$ & - & - & - \\
\hline Legatus leucophaius & Piratic Flycatcher & - & - & S & 2 & - \\
\hline Mionectes oleagineus & Ochre-bellied Flycatcher & - & $\mathrm{U}$ & $\mathrm{S}$ & - & 2 \\
\hline Miarchus ferox & Short-crested Flycatcher & - & - & $\mathrm{S}$ & - & 3 \\
\hline
\end{tabular}

Conservation status is described as NT (near threatened), V (vulnerable), E (endangered). GS (Guiana Shield) refers to a regional endemic. Abundance is recorded as $\mathrm{C}$ (common), more than 20 individuals encountered daily in prime habitat and season; $\mathrm{F}$ (fairly common) 5-20 individuals encountered daily in prime habitat and season; U (uncommon), fewer than 5 individuals encountered, not encountered daily even in prime habitat and season; S (scarce) only occasionally encountered in small numbers even in prime habitat and season; * Recorded present above Corona Falls, but not recorded during a survey.

O status da conservação está descrito como NT (ameaçada), V (vulnerável), E (em perigo). GS (Escudo da Guiana) se refere à região de endemismos. A abundância foi registrada como C (comum), quando mais de 20 indivíduos foram encontrados diariamente em seu habitat original e estação ótimos; $\mathrm{F}$ (relativamente comum) 5-20 indivíduos encontrados diariamente em seu habitat e estação ótimos; U (incomum) menos de 5 indivíduos encontrados; não encontrados diariamente mesmo no habitat original e estação ótimos; S (raro) apenas ocasionalmente encontrados em pequeno número mesmo no habitat original e estação ótimos. * Indivíduos encontrados acima Corona Falls, mas não encontrados durante um exame. 
Table 2. Continued...

Tabela 2. Continuação...

\begin{tabular}{|c|c|c|c|c|c|c|}
\hline \multirow{2}{*}{$\begin{array}{c}\text { Families and } \\
\text { species }\end{array}$} & \multirow{2}{*}{$\begin{array}{c}\text { Common } \\
\text { name }\end{array}$} & \multirow{2}{*}{$\begin{array}{c}\text { Conservation } \\
\text { and } \\
\text { endemism }\end{array}$} & \multirow{2}{*}{$\begin{array}{c}\text { SI } \\
\text { expedition } \\
\text { Aug 2006 } \\
\text { abundance }\end{array}$} & \multicolumn{3}{|c|}{ ZSL expedition Jan./2009 } \\
\hline & & & & Abundance & $\begin{array}{c}\text { Recorded } \\
\text { during } \\
\text { river counts }\end{array}$ & $\begin{array}{c}\text { Caught } \\
\text { in mist } \\
\text { nets }\end{array}$ \\
\hline Colonia colonus & Long-tailed Tyrant & - & - & $\mathrm{S}$ & 2 & - \\
\hline Ochthornis littoralis & Drab Water Tyrant & - & - & $\mathrm{F}$ & 80 & - \\
\hline Zimmerius gracilipes & Slender-footed Tyrannulet & - & $S$ & - & - & - \\
\hline Lophotriccus vitiosus & $\begin{array}{l}\text { Double-banded } \\
\text { Pygmy-Tyrant }\end{array}$ & - & $\mathrm{F}$ & - & - & - \\
\hline Lophotriccus galeatus & Helmeted Pygmy-Tyrant & - & $\mathrm{U}$ & $\mathrm{U}$ & 5 & - \\
\hline Myiornis ecuadatus & Short-tailed Pygmy-Tyrant & - & & $\mathrm{F}$ & 18 & - \\
\hline Todirostrum pictum & Painted Tody-Flycatcher & - & $\mathrm{U}$ & - & - & - \\
\hline Todirostrum cinereum & Common Tody-Flycatcher & - & - & $\mathrm{S}$ & 1 & - \\
\hline Corythopis torquata & Ringed Antpipit & - & $\mathrm{U}$ & - & - & - \\
\hline Tolmomyias assimilis & Yellow-margined Flycatcher & - & $\mathrm{F}$ & - & - & - \\
\hline Platyrinchus coronatus & Golden-crowned Spadebill & - & $\mathrm{F}$ & $\mathrm{F}$ & - & 9 \\
\hline Platyrinchus platyrhynchos & White-crested Spadebill & - & $\mathrm{S}$ & - & - & - \\
\hline Platyrinchus saturatus & Cinnamon-crested Spadebill & - & $S$ & - & - & - \\
\hline Onychorhynchus coronatus & Royal Flycatcher & - & $\mathrm{U}$ & - & - & - \\
\hline Myiobius barbatus & Sulphur-rumped Flycatcher & - & $\mathrm{U}$ & - & - & - \\
\hline Attila spadiceus & Bright-rumped Attila & - & $\mathrm{U}$ & $\mathrm{S}$ & 3 & - \\
\hline Attila cinnamomeus & Cinnamon Attila & - & - & $\mathrm{S}$ & 1 & - \\
\hline Rhytipterna simplex & Grayish Mourner & - & $\mathrm{U}$ & - & - & - \\
\hline Myiozetetes luteiventris & Dusky-chested Flycatcher & - & $\mathrm{U}$ & - & - & - \\
\hline Myiozetetes cayanensis & Rusty-margined Flycatcher & - & - & $\mathrm{F}$ & 21 & - \\
\hline Conopias parva & Yellow-throated Flycatcher & - & $\mathrm{F}$ & $\mathrm{U}$ & 8 & - \\
\hline Tyrannus melancholicus & Tropical Kingbird & - & $\mathrm{U}$ & $\mathrm{F}$ & 17 & - \\
\hline Pitangus lictor & Lesser Kiskadee & - & - & $\mathrm{U}$ & 7 & - \\
\hline Schiffornis turdinus & Thrush-like Schiffornis & - & $\mathrm{F}$ & $\mathrm{S}$ & 3 & 1 \\
\hline Lipaugus vociferans & Screaming Piha & - & $\mathrm{F}$ & $\mathrm{C}$ & 145 & - \\
\hline Laniocera hypopyrra & Cinereous Mourner & - & $\mathrm{U}$ & - & - & - \\
\hline Pachyramphus marginatus & Black-capped Becard & - & $\mathrm{U}$ & - & - & - \\
\hline $\begin{array}{l}\text { Pachyramphus minor } \\
\text { COTINGIDAE }\end{array}$ & Pink-throated Becard & - & $S$ & - & - & - \\
\hline Xipholena punicea & Pompadour Cotinga & - & S & $\mathrm{S}$ & 3 & - \\
\hline Perissocephalus tricolor & Capuchinbird & - & $\mathrm{U}$ & $\mathrm{S}$ & 1 & - \\
\hline Phoenicircus carnifex & Guianan Red Cotinga & - & - & $\mathrm{S}$ & 1 & - \\
\hline Cotinga cayana & Spangled Cotinga & - & - & $\mathrm{S}$ & 1 & - \\
\hline $\begin{array}{l}\text { Querula purpurata } \\
\text { TITYRIDAE }\end{array}$ & Purple-throated Fruitcrow & - & - & $\mathrm{S}$ & 1 & - \\
\hline $\begin{array}{l}\text { Tityra cayana } \\
\text { PIPRIDAE }\end{array}$ & Black-tailed Tityra & - & - & $\mathrm{S}$ & 1 & - \\
\hline Pipra pipra & White-crowned Manakin & - & $\mathrm{F}$ & $S$ & - & 2 \\
\hline Pipra erythrocephala & Golden-headed Manakin & - & $\mathrm{F}$ & - & - & - \\
\hline Tyranneutes virescens & Tiny Tyrant-Manakin & - & $\mathrm{U}$ & $\mathrm{U}$ & 6 & - \\
\hline Manacus manacus & White-bearded Manakin & - & - & $\mathrm{S}$ & 1 & - \\
\hline VIREONIDAE & & & & & & \\
\hline Hylophilus muscicapinus & Buff-cheeked Greenlet & - & $\mathrm{F}$ & - & - & - \\
\hline
\end{tabular}

Conservation status is described as NT (near threatened), V (vulnerable), E (endangered). GS (Guiana Shield) refers to a regional endemic. Abundance is recorded as $\mathrm{C}$ (common), more than 20 individuals encountered daily in prime habitat and season; F (fairly common) 5-20 individuals encountered daily in prime habitat and season; $\mathrm{U}$ (uncommon), fewer than 5 individuals encountered, not encountered daily even in prime habitat and season; $\mathrm{S}$ (scarce) only occasionally encountered in small numbers even in prime habitat and season; * Recorded present above Corona Falls, but not recorded during a survey.

O status da conservação está descrito como NT (ameaçada), V (vulnerável), E (em perigo). GS (Escudo da Guiana) se refere à região de endemismos. A abundância foi registrada como C (comum), quando mais de 20 indivíduos foram encontrados diariamente em seu habitat original e estação ótimos; F (relativamente comum) 5-20 indivíduos encontrados diariamente em seu habitat e estação ótimos; U (incomum) menos de 5 indivíduos encontrados; não encontrados diariamente mesmo no habitat original e estação ótimos; S (raro) apenas ocasionalmente encontrados em pequeno número mesmo no habitat original e estação ótimos. * Indivíduos encontrados acima Corona Falls, mas não encontrados durante um exame. 
Table 2. Continued...

Tabela 2. Continuação...

\begin{tabular}{|c|c|c|c|c|c|c|}
\hline \multirow{2}{*}{$\begin{array}{c}\text { Families and } \\
\text { species }\end{array}$} & \multirow{2}{*}{$\begin{array}{c}\text { Common } \\
\text { name }\end{array}$} & \multirow{2}{*}{$\begin{array}{l}\text { Conservation } \\
\text { and } \\
\text { endemism }\end{array}$} & \multirow{2}{*}{$\begin{array}{c}\text { SI } \\
\text { expedition } \\
\text { Aug 2006 } \\
\text { abundance }\end{array}$} & \multicolumn{3}{|c|}{ ZSL expedition Jan./2009 } \\
\hline & & & & Abundance & $\begin{array}{l}\text { Recorded } \\
\text { during } \\
\text { river counts }\end{array}$ & $\begin{array}{c}\text { Caught } \\
\text { in mist } \\
\text { nets }\end{array}$ \\
\hline Cyclarhis gujanensis & Rufous-browed Peppershrike & - & - & $\mathrm{U}$ & 10 & - \\
\hline Vireolaneus leucotis & Slatey-capped Shrike-Vireo & - & - & $\mathrm{U}$ & 8 & - \\
\hline \multicolumn{7}{|l|}{ HIRUNDINIDAE } \\
\hline Tachycineta albiventer & White-winged Swallow & - & $\mathrm{F}$ & $\mathrm{C}$ & 136 & - \\
\hline Atticora fasciata & White-banded Swallow & - & $\mathrm{C}$ & $\mathrm{C}$ & 735 & - \\
\hline Progne chalybea & Grey-breasted martin & - & - & $\mathrm{S}$ & 3 & - \\
\hline \multicolumn{7}{|l|}{ TROGLODYTIDAE } \\
\hline Henicorhina leucosticta & White-breasted Wood-Wren & - & $\mathrm{S}$ & - & - & - \\
\hline Thryothorus coraya & Coraya Wren & - & $\mathrm{U}$ & $\mathrm{U}$ & 13 & - \\
\hline Thryothorus leucotis & Buff-breasted Wren & - & $\mathrm{U}$ & $\mathrm{F}$ & 29 & - \\
\hline Microcerculus bambla & Wing-banded Wren & - & $\mathrm{S}$ & - & - & - \\
\hline Cyphorhinus arada & Musician Wren & - & $\mathrm{S}$ & $\mathrm{S}$ & 1 & - \\
\hline SYLVIIDAE & & & & & & \\
\hline $\begin{array}{l}\text { Ramphocaenus melanurus } \\
\text { TURDIDAE }\end{array}$ & Long-billed Gnatwren & - & $\mathrm{S}$ & - & - & - \\
\hline Turdus albicollis & White-necked Thrush & - & $\mathrm{U}$ & - & - & - \\
\hline Turdus fumigatus & Cocoa Thrush & - & - & $\mathrm{S}$ & 4 & - \\
\hline \multicolumn{7}{|l|}{ PARULIDAE } \\
\hline $\begin{array}{l}\text { Phaeothlypis rivularis } \\
\text { COEREBIDAE }\end{array}$ & River Warbler & - & $S$ & - & - & - \\
\hline $\begin{array}{l}\text { Coereba flaveola } \\
\text { THRAUPIDAE }\end{array}$ & \multicolumn{4}{|c|}{ THRAUPIDAE } & 1 & - \\
\hline Lanio fulvus & Fulvous Shrike-Tanager & - & $?$ & - & - & - \\
\hline Tachyphonus surinamus & Fulvous-crested Tanager & - & $\mathrm{U}$ & $\mathrm{S}$ & $*$ & - \\
\hline Ramphocelus carbo & Silver-beaked Tanager & - & $\mathrm{U}$ & $\mathrm{F}$ & 30 & - \\
\hline Thraupis episcopus & Blue-gray Tanager & - & & & & - \\
\hline Tangara mexicana & Turquoise Tanager & - & $\mathrm{U}$ & $\mathrm{S}$ & 3 & - \\
\hline Tangara velia & Opal-rumped Tanager & - & - & $\mathrm{S}$ & 4 & - \\
\hline Dacnis cayana & Blue Dacnis & - & $\mathrm{S}$ & - & - & - \\
\hline Tersina viridis & Swallow Tanager & - & - & $\mathrm{U}$ & 28 & - \\
\hline \multicolumn{7}{|l|}{ EMBERIZINAE } \\
\hline $\begin{array}{l}\text { Paroaria gularis } \\
\text { CARDINALINAE }\end{array}$ & Red-capped Cardinal & - & $\mathrm{U}$ & - & - & - \\
\hline Pitylus grossus & Slate-colored Grosbeak & - & $\mathrm{F}$ & $\mathrm{U}$ & 11 & - \\
\hline Saltator coerulescens & Greyish Saltator & - & $\mathrm{S}$ & $\mathrm{S}$ & 1 & - \\
\hline Caryothraustes canadensis & Yellow-green Grosbeak & - & $\mathrm{F}$ & - & - & - \\
\hline Cyanocompsa cyanoides & Blue-black Grosbeak & - & $\mathrm{U}$ & $\mathrm{S}$ & 3 & - \\
\hline FRINGILIDAE & & & & & & \\
\hline $\begin{array}{l}\text { Euphonia violacea } \\
\text { ICTERIDAE }\end{array}$ & \multicolumn{5}{|c|}{ ICTERIDAE } & - \\
\hline Scaphidura oryzivora & Giant Cowbird & - & - & $\mathrm{F}$ & 32 & - \\
\hline Molothrus bonariensis & Shiny Cowbird & - & - & $\mathrm{U}$ & 5 & - \\
\hline Cacicus cela & Yellow-rumped Cacique & - & $\mathrm{U}$ & $\mathrm{C}$ & 60 & - \\
\hline Cacicus haemorrhous & Red-rumped Cacique & - & - & $\mathrm{C}$ & 29 & - \\
\hline Psarocolius decumanus & Crested Oropendola & - & $\mathrm{U}$ & $\mathrm{C}$ & 64 & - \\
\hline Psarocolius viridis & Green Oropendola & - & $\mathrm{U}$ & $\mathrm{U}$ & 8 & - \\
\hline \multicolumn{7}{|c|}{$\begin{array}{l}\text { Conservation status is described as NT (near threatened), V (vulnerable), E (endangered). GS (Guiana Shield) refers to a regional endemic. Abundance is } \\
\text { recorded as C (common), more than } 20 \text { individuals encountered daily in prime habitat and season; F (fairly common) } 5 \text {-20 individuals encountered daily } \\
\text { in prime habitat and season; U (uncommon), fewer than } 5 \text { individuals encountered, not encountered daily even in prime habitat and season; S (scarce) only } \\
\text { occasionally encountered in small numbers even in prime habitat and season; * Recorded present above Corona Falls, but not recorded during a survey. }\end{array}$} \\
\hline $\begin{array}{l}\text { O status da conservação está descrit } \\
\text { A abundância foi registrada como C } \\
\text { (relativamente comum) 5-20 indivídu } \\
\text { encontrados diariamente mesmo no } \\
\text { original e estação ótimos. * Indivídu }\end{array}$ & $\begin{array}{l}\text { no NT (ameaçada), V (vulneráve } \\
\text { um), quando mais de } 20 \text { indivíd } \\
\text { ncontrados diariamente em seu h } \\
\text { t original e estação ótimos; S (ra }\end{array}$ & $\begin{array}{l}\text { l), E (em perigo) } \\
\text { uos foram encontı } \\
\text { abitat e estação ó }\end{array}$ & $\begin{array}{l}\text { GS (Escudo da } \\
\text { dos diariament } \\
\text { mos; U (incom }\end{array}$ & $\begin{array}{l}\text { Guiana) se refer } \\
\text { em seu habitat } \\
\text { m) menos de } 5\end{array}$ & $\begin{array}{l}\text { re à região de e } \\
\text { original e estaçã } \\
\text { indivíduos enco }\end{array}$ & $\begin{array}{l}\text { demismos. } \\
\text { o ótimos; F } \\
\text { trados; não }\end{array}$ \\
\hline
\end{tabular}



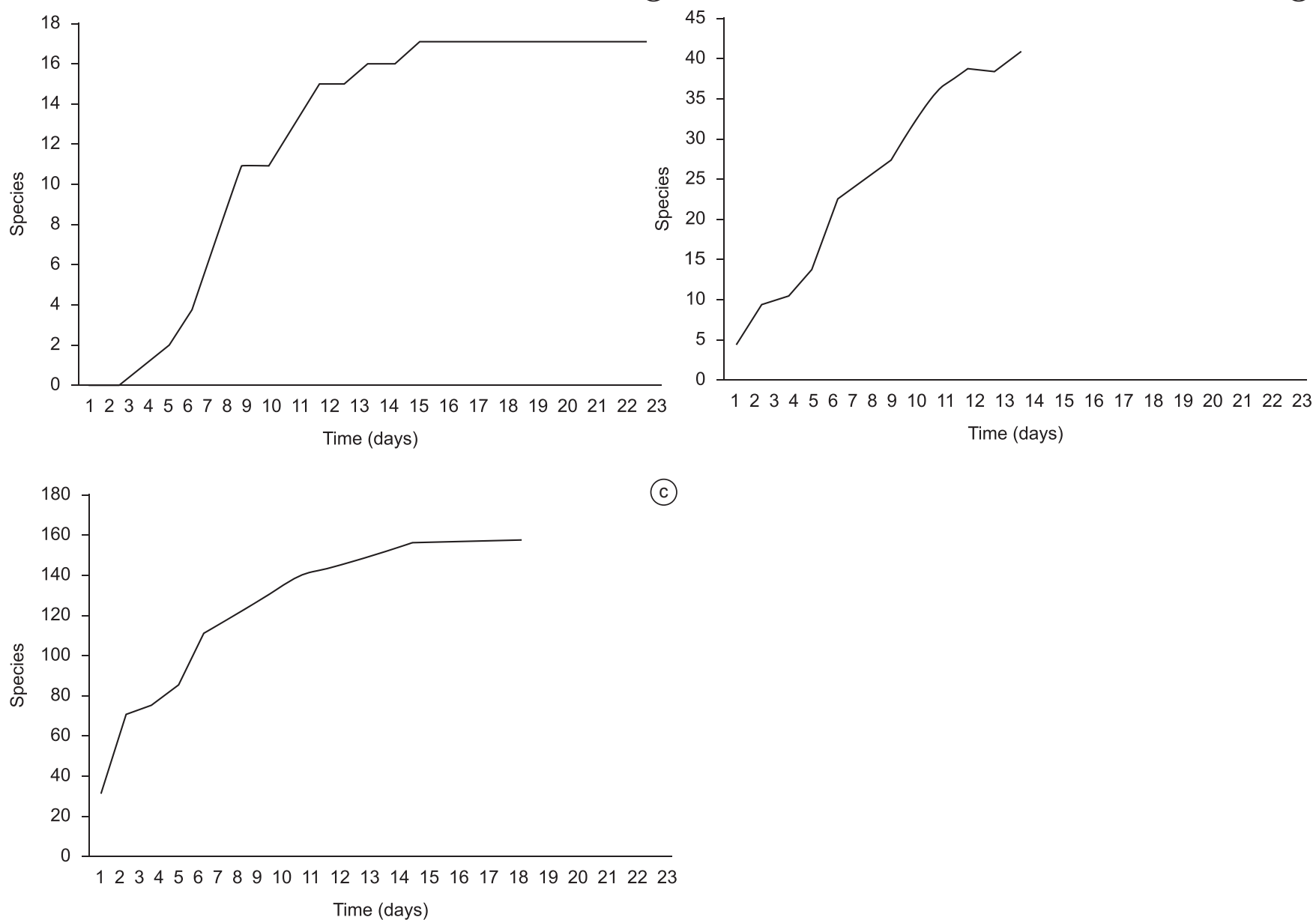

Figure 4. Species accumulation curves: a) camera trap survey (top left), b) mist net survey (top right), c) drift spot count survey (bottom).

Figura 4. As curvas de acúmulo de espécies: a) armadilha fotográficas (canto superior esquerdo), b) redes de neblina (acima à direita), c) avistamentos do aves (em baixo).

to be important for jaguar (Panthera onca): three were recorded during our expedition and numerous faecal deposits were also encountered. Holland recorded 11 daylight jaguar sightings over six weeks during a period of extreme low water in 2006. The difficulty of portaging boats above the falls into the Rewa Head has prevented the expansion of hunters into the area. Consequently game species such as lowland tapir (Tapirus terrestris), paca (Agouti paca) and black curassow (Crax alector) are both common and naïve. During the course of the expedition four tapirs were encountered in the river during the day and tolerated us approaching to within $5 \mathrm{~m}$.

The presence of the bush dog is also important. The bush dog is an elusive and poorly understood animal, with most data on its behaviour and diet derived from anecdotes. In one study on diet in the Brazilian Pantanal, de Souza Lima et al. (2009) recorded that the predominant prey found in faeces was the nine-banded long-nosed armadillo (Dasypus novemcinctus), which appears abundant in the Rewa Head. Although its range is large and it is found throughout Amazonia, it is considered to occur in low densities. The IUCN red list classifies the species as 'Near Threatened' being likely to suffer a $10 \%$ decline over the following decade due to habitat degradation (Zuercher et al. 2008).

The density of the giant otter is similar to that recorded on the Rio San Martin in Bolivia by Zambrana Rojas (2007). Following the cessation of commercial hunting, these headwaters of the River
Rewa likely constituted a source for giant otter expansion within Guyana. While there is no commercial hunting of giant otters, they are occasionally persecuted due to the perceived competition with fishermen, and suffer from habitat loss and disturbance due to gold mining (Groenendijk et al. 2005).

The demands of resource extraction in Southern Guyana in the near future may lead to conflict with the interests of conservation of biodiversity. The Rewa Head constitutes part of a 400,000 ha logging concession (Simon \& Shock International 2007). While there has recently been a moratorium put in place on gold dredging in the Rewa, the security of the rivers from wildcat miners is in doubt.

\section{Conclusion}

River surveys were conducted alongside to the secondary goal of searching for sign of giant otter activity. Such surveys are biased towards riparian species of bird and further expeditions should also include spot-count transects within the forest itself. Small mammal trapping and bat netting were also not conducted and yet these surveys as well as extensive ichthyological surveys are extremely important for determining the community assemblage of this important forest, especially in light of the recent identification of 86 species of bat in the Iwokrama reserve alone (the highest of any protected area), and of the identification of a high degree of endemism in the aquatic fauna of the Essequibo drainage basin. The conservation potential of the Rewa 
Head is high in that it lies between the Upper Essequibo Concession, maintained by Conservation International and the proposed Kanuku Mountains Protected Area (Montambaut \& Missa 2002). Protection of the Rewa Head would form a protected area spanning almost the width of Guyana. We conclude from our results that the Rewa Head is an ecologically important area within the Guiana Shield rainforest and merits recognition, and that further studies exploring the biological richness of the region are recommended.

\section{Acknowledgements}

Thanks to Chris Milensky and Brian Schmidt and the Smithsonian Institute for the use of their unpublished 2006 data. Many thanks go to Diane McTurk for help and advice. Thanks to Margaret Chan-a-sue for logistical support in Georgetown and to Peter Taylor for sound advice. Many thanks to Cristina Ariani for the Portuguese translations and thanks to the reviewers of this paper for helpful comments. This expedition was funded through generous grants from the Linnaean Society's Percy Sladen Memorial Foundation, ZSL's Daisy Balogh Travel Award and through NERC expedition funds.

\section{References}

ALONSO, L.E., McCULLOUGH, J., NASKRECKI, P., ALEXANDER, E., \& WRIGHT, H.E. 2008. A rapid biological assessment of the Konashen Community Owned Conservation Area, Southern Guyana. RAP Bulletin of Biological Assessment 51. Conservation International, Arlington, VA, USA.

BRAUN, M.J., FINCH, D.W., ROBBINS, M.B., \& SCHMIDT, B.K. 2007. A Field Checklist of the Birds of Guyana. Centre for the Study of Biological Diversity, University of Guyana, Georgetown, Guyana; Biological Diversity of the Guianas Program, National Museum of Natural History, Smithsonian Institute, Washington.

BEAIE, S.T. 2002.The Cooperative Republic of Guyana population and housing census 2002. Bureau of Statistics Guyana.

EMMONS, L.H., \& FEER, F. 1997. Neotropical Rainforest Mammals, A Field Guide. 2nd ed. University of Chicago Press, Chicago.

ENGSTROM, M. \& LIM, B. 2008. Checklist of the Mammals of Guyana. Disponível em http://www.mnh.si.edu/biodiversity/bdg/guymammals. html (último acesso em 21/01/2011).

DE SOUZA LIMA, E., JORGE, R. S. P., DALPONTE, J. C. 2009. Habitat use and diet of bush dogs, Speothos venaticus, in the Northern Pantanal, Mato Grosso, Brazil. Mammalia 73: 13-19.

DUPLAIX, N., WALDEMARIN, H.F., GROENENDIJK, J., EVANGELISTA, E., MUNIS, M., VALESCO, M., BOTELLO, J.C. 2008. Pteronura brasiliensis. In IUCN Red List of Threatened Species (International Union for Conservation of Nature - IUCN). Version 2010.4. Disponível em www.iucnredlist.org (último acesso em 12/05/2011).

GROENENDIJK, J., HAJEK, F., DUPLAIX, N., REUTHER, C., DAMME, P. van, SCHENCK, C., STAIB, E., WALLACE, R., WALDEMARIN, H., NOTIN, R., MARMONTEL, M., ROSAS, F., MATTOS, G.E., EVANGELISTA, E., UTRERAS, V., LASSO, G., JACQUES, H., MATOS, K., ROOPSIND, I. \& BOTELLO, J.C. 2005. Surveying and monitoring distribution and population trends of the giant otter (Pteronura brasiliensis). Guidelines for a standardisation of survey methods as recommended by the giant otter section of the IUCN/SSC Otter Specialist Network. Habitat 16:1-100.
GUYANA FORESTRY COMMISSION. 2007. Factsheet. Disponível em http://www.forestry.gov.gy/Downloads/Fact\%20Sheet\%202007.pdf (último acesso em 27/09/2010).

HAJEK, F., GROENENDIJK. J., SCHENCK, C., STAIB, E., 2005. Population census methodology guidelines for the giant otter (PCMG-GO). In (J. Groenendijk, F. Hajek, N. Duplaix, C. Reuther, P.van Damme, C. Schenck, E. Staib, R. Wallace, H. Waldemarin, R. Notin, M. Marmontel, F. Rosas, G.E. Mattos, E. Evangelista, V. Utreras, G. Lasso, H. Jacques, K. Matos, I. Roopsind \& J.C. Botello). Surveying and monitoring distribution and population trends of the giant otter (Pteronura brasiliensis). Guidelines for a standardisation of survey methods as recommended by the giant otter section of the IUCN/SSC Otter Specialist Network. Habitat 16:1-100.

HAMMOND, D.S., ed. 2005. Tropical Forests of the Guiana Shield. CABI Publishing, Oxford.

HILTY, S. 2002. Birds of Venezuela. Christopher Helm, London.

IUCN 2008. IUCN Red List of Threatened Species. Version 2008.1. <http:// www.iucnredlist.org>. Downloaded on 16 June 2008.

LIBRARY OF CONGRESS - LOC. 1992. Guyana Country Study. Library of Congress, Washington.

LIM, B.K \& ERNGSTROM, M.D. 2001. Species diversity of bats (Mammalia: Chiroptera) in Iwokrama Forest, Guyana, and the Guianan subregion: implications for conservation. Biodivers. Conserv. 10:613-657. http:// dx.doi.org/10.1023/A:1016660123189

MILENSKY, C.M. \& SCHMIDT, B.K. 2006. Upper Rewa River Bird Checklist. Smithsonian Institution Expedition.

LIM, B.K. \& ENGSTROM, M.D. 2004. Mammals of Iwokrama Forest. Proc. Acad. Nat. Sci. Philadelphia 154:71-108. http://dx.doi.org/10.1635/00973157(2004)154[0071:MOIF]2.0.CO;2

MILENSKY, C. M., HINDS, W., ALEIXO, A. \& LIMA, M.C. 2005. Birds. In Checklist of the Terrestrial Vertebrates of the Guiana Shield (T. Hollowell \& R.P. Reynolds, eds.). Bull. biol. Soc. Wash. (13):43-76. MILENSKY, C.M. \& SCHMIDT, B.K. 2006. Upper Rewa River Bird Checklist. Smithsonian Institution Expedition.

MONTAMBAUT, J.R. \& MISSA, O., eds. 2002. A Biodiversity Assessment of the Eastern Kanuku Mountains, Lower Kwitaro River, Guyana. Conservation International, Washington. (RAP Bulletin of Biological Assessment).

RESTALL, R., RODNER, C. \& LENTINO, M. 2006. Birds of Northern South America: An Identification Guide. Christopher Helm, London.

SAATCHI, S.S., HOUGHTON, R.A., DOS SANTOS ALVALA, R.C., SOARES, J.V. \& YU, Y. 2007. Diversity of above ground live biomass in the Amazon Basin. Glob. Chang. Biol. 13:816-837. http://dx.doi. org/10.1111/j.1365-2486.2007.01323.x

TOUCHTON, J.M., HSU, Y., \& PALLERONI, A. 2002. Foraging ecology of reintroduced captive-bred subadult harpy eagles (Harpia harpyja) on Barro Colorado Island, Panama. Ornitol. Neotrop. 13: 365-379.

ZAMBRANA ROJAS, V.D., 2007. Distribución y estado poblacional de la londra (Pteronura brasiliensis) en los ríos Blanco y San Martin (Cuenca del Rio Itenez). Undergratuate dissertation, Universidad Mayor de San Simón, Cochabamba.

ZUERCHER, G.L., SWARNER, M., SILVEIRA, L. \& CARILLO, O. 2008. Speothos venaticus. In IUCN Red List of Threatened Species (International Union for Conservation of Nature - IUCN). Disponível em www.iucnredlist.org (último acesso em 19/03/2009). 
Appendix 1. Mist-net survey sites.

N2 59.773 W58 35.971

Powys Camp

N3 07.901 W58 37.896

Split Camp

N2 37.752 W58 37.152

Tayra Camp

N2 45.358 W58 37.415

Onca Camp

N2 53.697 W58 35.225 